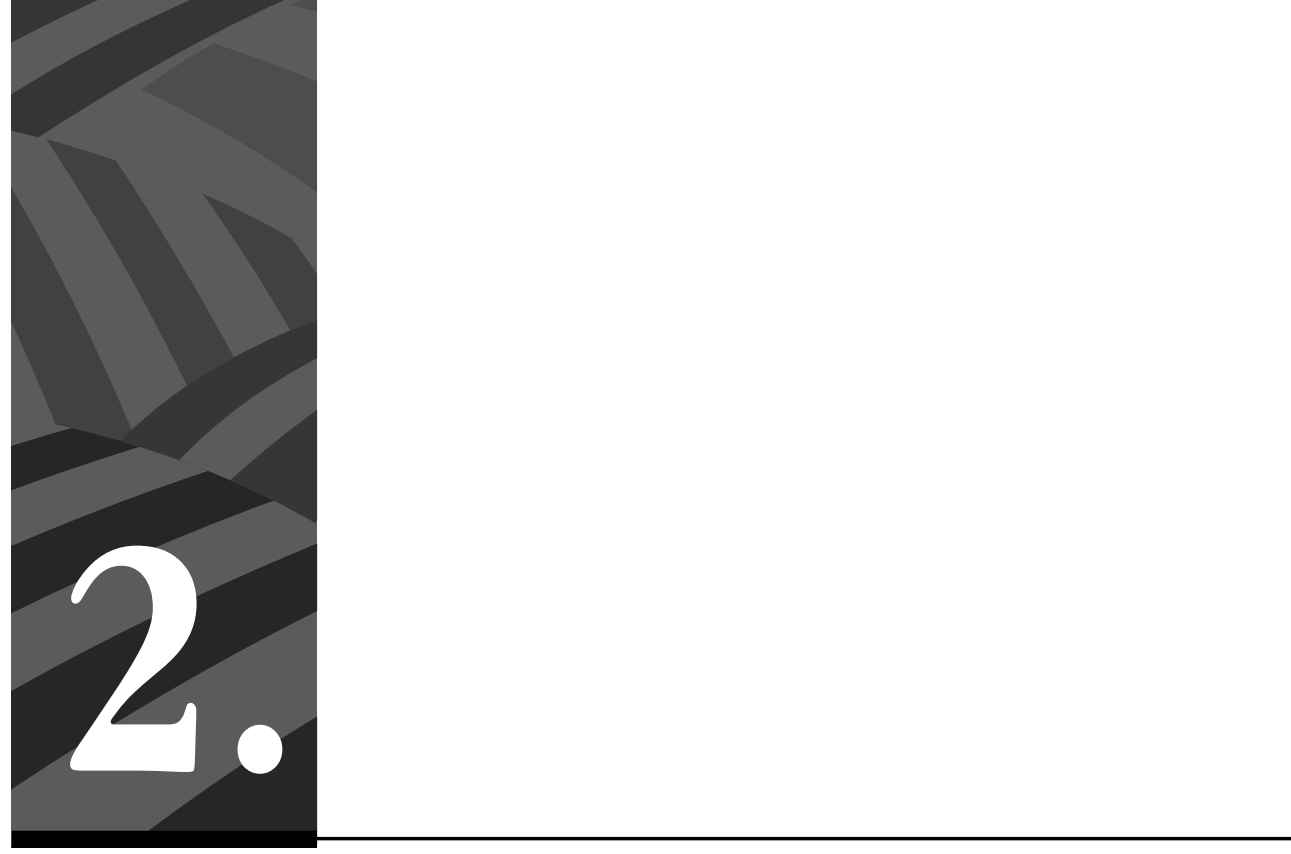

Gestar y abortar para los hombres trans: una revisión de literatura científica 


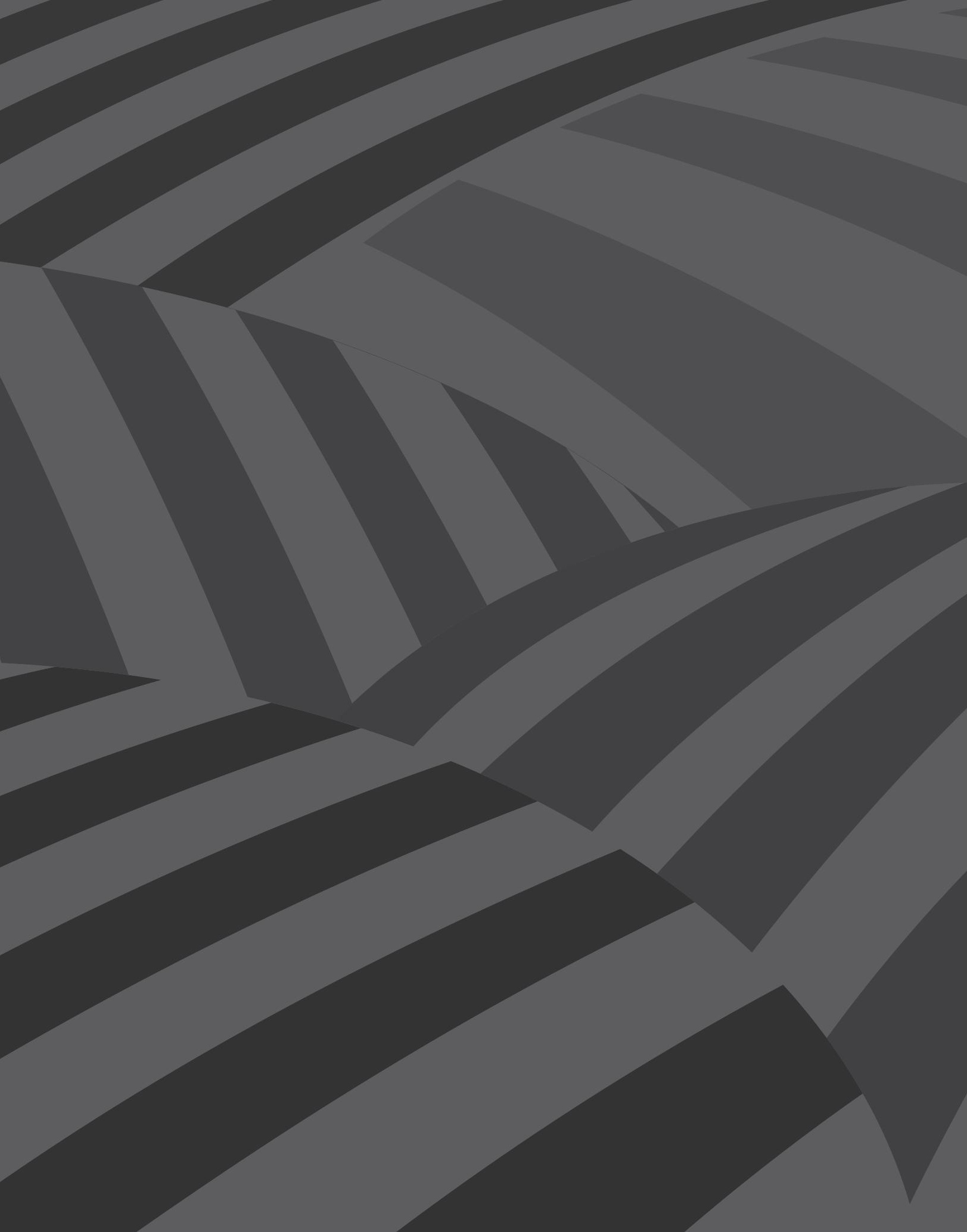




\title{
Gestar y abortar para los hombres trans: una revisión de literatura científica
}

\author{
Por María Antonella Barone*
}

Resumen: En este artículo se presenta una revisión de las investigaciones disponibles en la literatura sobre procesos de gestación y aborto de los hombres trans, a partir de la búsqueda en bases de datos como PsyeINFO y Scielo, de términos normalizados como pregnancy, aborto, trans men, transgender, transexual. Tal revisión de literatura es parte de mi tesis de doctorado, aun no concluida, titulada "Narrativas transbordantes: experiencias de personas trans, a partir de prácticas de gestación y aborto”, en el marco de una posgraduación en el área de Psicología. En consecuencia, se seleccionan publicaciones que consideran aspectos psicológicos o sociales sobre el tema, con abordajes desde el área de la salud hasta estudios más próximos a las ciencias humanas y sociales, con el fin de identificar los estudios relativos a esta temática. Se observa, de este modo, que la mayoría de las referencias son de los últimos diez años. Sin embargo, cabe destacar un déficit de investigaciones sobre aborto y hombres trans.

Palabras clave: gestación, aborto, hombres trans, transexualidad.

\section{Gestating and Aborting for Trans Men: A Review of Scientific Literature}

Abstract: In this article, we present a review of the research available in the literature on gestation and abortion processes in trans men, based on a search in databases such as PsyeINFO and Scielo, for standardized terms such as pregnancy, abortion, trans men, transgender, tranny. This literature review is part of my doctoral thesis, not yet completed, entitled "Transborder Narratives: experiences of trans people, from pregnancy and abortion practices", within the framework of a Postgraduate degree in the area of Psychology. In this way, publications are selected that consider psychological or social aspects of the subject, with approaches from the health area to studies closer to the human and social sciences, in order to identify studies related to this subject. In this way, it is

* Graduación en Psicología por la Universidad Nacional de Córdoba, Argentina. Maestría en Psicología Institucional por la Universidade Federal do Espirito Santo, Brasil. Doctoranda en Psicología por la Universidade Federal do Espírito Santo, Brasil. Investigadora responsable por el Grupo de Estudos sobre Aborto perteneciente al Núcleo de Estudos e Pesquisas em Gênero e Sexualidades. Correo electrónico: licenciadaantonellabarone@gmail.com. 
observed that most of the references are from the last ten years. However, it is worth noting a lack of research on abortion and trans men.

Keywords: pregnancy; abortion; trans men; transsexuality.

Cómo citar este artículo: Antonella Barone, María (2020). Gestar y abortar para los hombres trans: una revisión de literatura científica. Revista Controversia, 215, 43-73.

Fecha de recepción: 22 de marzo de 2020

Fecha de aprobación: 17 de julio de 2020

\section{Introducción}

T os resultados de esta revisión de literatura científica permiten acceder a un conocimiento actualizado en torno de términos como pregnancy, aborto, abortion, trans men, transgender, transexual, referidos a las experiencias de las personas trans, en cuanto a su carácter de personas gestantes. Dicha revisión fue realizada en las bases PsycINFO y Scielo y, particularmente, en las investigaciones que abordan las prácticas de aborto de los hombres trans. En ese propósito, se considera relevante para la coyuntura de Latinoamérica y el Caribe conocer las investigaciones más recientes sobre las políticas en materia de aborto de las personas trans.

$\mathrm{Al}$ efecto, se seleccionaron publicaciones que consideraban aspectos psicológicos o sociales sobre el tema, siendo posible observar una diversidad de abordajes que iban desde estudios más ligados a la atención de la salud, de áreas como medicina y enfermería, a algunos estudios más próximos a las ciencias humanas y sociales. Se destaca, también, que las referencias son recientes, la mayoría de los últimos diez años. Sin embargo, y como problema cuya respuesta se busca obtener en este estudio, se considera necesario y urgente, inclusive en la agenda de los movimientos sociales, disminuir las brechas de investigación sobre los hombres trans y el aborto. Esto, para aportar al proceso de visibilización de las experiencias de las personas trans, en su carácter de personas gestantes y, por ende, pasibles de abortar. En este sentido es que se considera altamente preocupante el déficit de investigaciones relacionadas con el aborto vivenciado por personas trans. 


\section{Hombres trans: tonalidades de la compleja "acuarela de las masculinidades"}

Gestaciones, partos y abortos forman parte de las vivencias de las personas que componen una sociedad. Sin embargo, es notable la falta de información sobre las situaciones atravesadas por la población trans en sus prácticas de gestación y aborto. Con la expresión “población trans”, en este trabajo se refiere a aquellas personas que, a través de procesos de reinvención, "osan reivindicar una identidad de género en oposición a aquella informada por los genitales” (Bento, 2008, p. 22, traducción libre).

Es importante señalar que la apuesta ética y política de este trabajo persigue líneas de fuerza que consideran las dimensiones subjetivas de las personas trans. Es posible identificar que diferentes grupos y poblaciones difieren significativamente entre sí en la singularidad de sus experiencias y en las concepciones de su identidad. Se afirman estas diferencias y, de ningún modo, se intentará aquí una pretensión de universalización y homogeneidad de las trayectorias y multiplicidades de esta población. La adopción de una terminología específica tiene el objetivo pragmático de procurar una base conceptual que permita dilucidar los vacíos investigativos que existen, y aportar al direccionamiento de esfuerzos de construcción de herramientas de análisis para abordajes de la temática. Los términos y conceptos utilizados han de entenderse de manera flexible, a la luz de este objetivo.

Este estudio se interesa por conocer cuál es la producción científica sobre experiencias vivenciadas por los hombres trans, ya que en relación con las publicaciones sobre experiencias de gestación y aborto de las mujeres cis $^{1}$ existiría una tamaña desproporción. Como advierte Blas Radi (2018, Mito n. ${ }^{\circ}$ 10):

1 Se utiliza aquí el término cis para designar a las personas que no son trans. Es decir, se refiere a una designación de género que se mantiene en una cierta concordancia entre el asociado al sexo anatómico asignado antes de nacer (por instituciones médicas y jurídicas) y una identificación con ese género a partir de aquella designación a lo largo de la vida. Mujeres cis, entonces, serían aquellos cuerpos que nacieron con vagina y que, a lo largo de su vida, se identifican como mujeres. 
Hay que reconocer que todos los informes y estadísticas existentes sobre esta materia reflejan una desproporción elocuente: la relación entre mujeres cis y hombres trans relevados es de 100 a 0 . Por supuesto, cuando interrogamos acerca de los procedimientos para determinar estas cifras, los cálculos realizados y los datos brutos, encontramos que se trata de investigaciones que sólo se aplican a mujeres cis, entendiendo que son los únicos sujetos de embarazo, aborto, subrogación de vientres, etc. En esos casos, la inexistencia de hombres trans no es un resultado de la investigación sino una premisa. En este escenario, es necesario considerar que probablemente esa mayoría absoluta no sea más que una proyección sobre la realidad de limitaciones que pertenecen a los instrumentos de análisis.

En cualquier caso, incluso si existieran herramientas analíticas lo suficientemente sofisticadas como para registrar a los hombres trans, sería preciso considerar una serie de desafíos antes de volcarnos sobre los números. Por ejemplo, que en el marco de contextos hostiles es poco probable que las personas trans estén dispuestas a identificarse públicamente como tales, máxime teniendo en cuenta lo que se pone en juego en el espacio del consultorio médico. Ni hablar cuando se trata de declarar un aborto.

"Varones trans”, "hombres transexuales”, "transhombre”, "transman”, "FTM" (sigla en inglés de female-to-male, es decir, femenino para masculino, utilizada en la literatura médica) son variaciones en los modos de referirse a experiencias de transexualidad masculina. En este artículo, y para evitar un uso de múltiples expresiones, se utiliza la vocablo "hombre trans" como un criterio de nombramiento, tratándose más de una categoría práctica “de reconocimiento que de categorías rígidas para el análisis científico o de taxonomías que surgen exclusivamente de las instituciones de poder que patologizan, definen y clasifican la diferencia” (García Becerra, 2009, p. 121). Es decir, el acto de adoptar la locución hombres trans sigue el único propósito de organizar este trabajo y, conforme presenta Guilherme Almeida (2012), mostrar solo algunas tonalidades de la compleja "acuarela de las masculinidades" que caracteriza a los sujetos a quienes se les asignó una producción de femineidad desde antes de nacer, pero que, de formas diversas y variables, se oponen a esa designación. 


\section{Carácter gestante de los hombres trans: posibilidades y experiencias}

Cuando un óvulo es fertilizado por un espermatozoide comienza un proceso denominado gestación. A partir de allí, como mínimo, se desprenden dos posibilidades de ese carácter gestante: una práctica de parto, cuando el ciclo gestacional se completa en todos sus estadios, o una práctica de aborto, cuando por diversos motivos se interrumpe la gestación.

Para una aproximación al carácter gestante de los hombres trans, se seleccionan algunas investigaciones sobre estas experiencias. Hoffkling, Obedin-Maliver y Sevelius (2017) presentan los resultados de un estudio de tipo cualitativo sobre las experiencias de los hombres trans en relación con sus gestaciones y establecen algunas recomendaciones para profesionales. Dicho estudio tuvo como objetivo comprender las necesidades de hombres trans que atravesaron por una práctica de parto. Para ello, entrevistaron a diez hombres trans que habían tenido una experiencia de parto mientras se identificaban como hombres, y realizaron un análisis de los temas centrales para la comprensión de estas experiencias. Al respecto, narraron diversas experiencias, incluyendo la priorización de la transición versus reproducción, deseo de afirmación externa de su identidad, deseo de afirmar la gestación, entre otras. Concluye, este estudio, que el reconocimiento de diversas experiencias tiene implicaciones en el apoyo a futuras atenciones de la salud y promueve cuidados centrados en el sujeto, desde los períodos de pretransición, preconcepción, prenatal y posparto. Así mismo, identifica formas de apoyar y destacar otras sensibilidades, para conocer sobre las experiencias de hombres trans. El estudio aclara que no investigaron las experiencias de hombres trans que decidieron no gestar nunca; de aquellos que deseaban una gestación, pero no tenían esa capacidad en sentido biológico; ni aquellos que gestaron, pero cuyas gestaciones se interrumpieron por aborto producido o espontáneo. 
Según las autoras, algunos hombres trans mantienen el útero, gestan y paren; sin embargo, las actitudes de la sociedad en relación con el género y la sexualidad crean algunas barreras. Así,

la materialidad del cuerpo debe ser analizada como efecto de un poder, y el sexo no son aquello que alguno tiene o una descripción estática. El sexo es una de las normas por las cuales la persona se torna viable, que cualifica un cuerpo para la vida en el interior del dominio de lo humano. (Bento, 2017, p. 71).

En sus trabajos sobre transexualidad, Berenice Bento realiza un abordaje del género como una maquinaria que produce cuerpos y subjetividades en serie y de manera binaria. Esta maquinaria funciona en la producción de cuerpos ajustados para caber en los moldes que ella misma fabrica, y, al mismo tiempo, provoca abyección hacia aquellos cuerpos que no se ajustan a la norma.

Esta producción puede observarse en algunos resultados de estudios que abordan las experiencias en relación con la gestación de hombres trans. La capacidad gestante fue investigada también en Australia (Charter, Ussher, Perz y Robinson, 2018). Participaron 25 hombres trans con edades entre 25 y 46 años. A partir de un análisis temático, se concluyó que los cambios relacionados con el retiro de testosterona y los cambios en el cuerpo, referidos principalmente a las alteraciones en los pechos, fueron vivenciados como una preocupación. Exclusión, aislamiento y soledad fueron las sensaciones predominantes de hombres trans durante la experiencia del embarazo, según este estudio. Generalmente encontraron problemas significativos al interactuar con profesionales del sistema de salud, que no los apoyaron. Estas dificultades se corresponderían con las concepciones edificadas, precisamente, por los saberes médicos y las áreas de la psicología que insisten en calificar a la transexualidad como una "enfermedad mental" relacionada con el campo de la sexualidad. A partir de la creación de trastornos, síndromes y los respectivos diagnósticos psiquiátricos atribuidos a esas 
experiencias, mediante la construcción de un conjunto de saberes, de técnicas y prácticas, se esfuerzan por explicar modos de sentir y pensar sexualidades y transexualidades (Bento, 2014).

Conocer y comprender el carácter gestante de las personas trans irrumpe como una necesidad, en la medida que confronta el modo hegemónico en que fueron producidos esos saberes patologizantes. Light, ObedinMaliver, Sevelius y Kerns (2014) investigaron sobre la experiencia de gestación de 41 hombres trans y encontraron que antes de la concepción, $61 \%$ usaron testosterona y $88 \%$ de los ovocitos provinieron de los propios participantes. La mitad de los participantes recibió atención por parte de un médico prenatal y $78 \%$ recibió atención durante el parto en un hospital. De las cuatro preguntas abiertas realizadas por dicha investigación, 30 participantes (73\%) respondieron por lo menos una. Los principales temas de esas respuestas estaban relacionados con: 1) efectos de la gestación en los conceptos de estructura familiar; 2) aislamiento; 3 ) cambios en el cuerpo y embarazo; e 4) interacciones con el personal profesional de salud.

En esta revisión de literatura se encontró otro estudio realizado en Canadá por Veale, Watson, Adjei y Saewyc (2016), con 923 participantes trans de entre 14 y 25 años de edad, con el propósito de explorar las experiencias de gestación y los factores de salud relacionados con la población trans. Los resultados indican que 26 de los jóvenes transexuales (5\%) narraron haber tenido una experiencia de gestación en el pasado. En respuesta a la pregunta sobre identidad de género, casi la mitad (48\%) relató identificarse como hombre, $36 \%$ se identificó como hombre trans, $8 \%$ como mujer y $12 \%$ como mujer trans. Esto muestra, una vez más, la necesidad de que profesionales de distintas disciplinas involucradas en la atención de las personas trans, se impliquen activamente con sus demandas específicas, principalmente aquellas provenientes de la salud sexual y (no) reproductiva. 
Ellis, Wojnar y Pettinato (2015) destacan en su estudio que la soledad fue el tema que permeabilizó las experiencias, interacciones sociales y respuestas emocionales de los participantes durante todas las etapas. En ese contexto, los participantes describieron complejos procesos de identidad, incluyendo luchas internas e interaccionales con el mundo. El periodo de la preconcepción fue identificado por los participantes como el momento de mayor sufrimiento y de menor cuidado con la salud. La soledad fue un tema que atravesó los periodos de preconcepción, prenatal y posparto, según la investigación. Las respuestas a los cambios físicos del embarazo fueron variadas, bienvenidas o no. Los puntos de tensión frente al sistema de salud incluyeron incomodidades y miedo en la relación con los profesionales tanto al comunicarse con el equipo médico como al recibir atención hospitalaria respecto a las necesidades específicas de esta población.

Un tema que reúne varias publicaciones y ocupa un lugar destacado en la producción científica es el que se refiere al mantenimiento de la fertilidad de los hombres trans. Johnson y Finlayson (2016) afirman que las personas trans enfrentan tratamientos médicos que pueden perjudicar su capacidad para gestar, y que la preservación de su fertilidad va a depender de las experiencias en sus singularidades. En ese sentido y de acuerdo con Blas Radi (2018) —quien cuestiona la mitología política que envuelve la problemática sobre el aborto y los hombres trans-, existen obstáculos que no son solo teóricos y políticos, sino también culturales, en el abordaje de las situaciones vividas por ellos en relación con experiencias sexuales y (no) reproductivas. En palabras del autor:

En rigor, algunos hombres trans desean tener hijxs y otros no. Como en cualquier grupo humano, ¿no? Una generalización tan burda debería caer por su propio peso. Sin embargo, es interesante prestarle atención, para analizar cómo funcionan los estereotipos normativos vinculados con el género y la reproducción. Estos estereotipos permean la cultura y su funcionamiento cotidiano constituye las condiciones de posibilidad de los cuerpos generizados. (...) Muchas mujeres cis son estériles y no diríamos 
por eso que ninguna puede procrear. ¿Qué habilita la inducción cuando se trata de hombres trans? Tal vez sea el flagelo de la esterilización forzada. Es un buen punto. La renuncia a las capacidades reproductivas ha sido y es condición de acceso al reconocimiento legal de la identidad de género de las personas trans en muchos países. En algunos casos, la esterilización forzada es uno de los requisitos establecidos por la ley o los tribunales, aunque no es exigida abiertamente. (Radi, 2018, Mito n. ${ }^{\circ} 2$ ).

Pese a las imposiciones institucionales aún vigentes en muchos países donde es el Estado el que restringe determinados modos de vivir la sexualidad y la procreación, multiplicidad de experiencias se colocan como posibles en el carácter gestante de las personas trans. Aun así, habiendo hombres trans que han gestado y parido, "en muchos Estados, la renuncia a las capacidades reproductivas es un requisito para el reconocimiento de una identidad de género distinta a la asignada al nacer” (Radi, 2018, Mito n. ${ }^{\circ} 2$ ).

Esto trae aparejada una serie de aspectos que envuelven dimensiones bio-psico-sociales, requiriendo delineamientos específicos para cada situación. Por ejemplo, el caso estudiado por De Castro-Peraza et al. (2019) se realizó a partir de una revisión de literatura sobre las personas trans, deseos de gestar y parir, y los aspectos biológicos, psicológicos, sociales y legales relacionados con esas experiencias. En su investigación se destaca el caso de un hombre trans que deseaba una gestación y que inició el ciclo de reproducción asistida cuando era reconocido legalmente como mujer cis, pero esa tentativa acabó en un aborto espontáneo. En el ciclo de reproducción asistida, 4 embriones permanecieron congelados. Luego de la experiencia de aborto, completó su transición y, una vez reconocido legalmente como hombre emprendió un proceso de gestación sin asistencia. En este proceso fueron planteados ampliamente los aspectos a conocer cuando se abordan gestaciones trans. Después de analizar las informaciones, la principal conclusión de ese estudio es que el deseo de gestar y parir, así como el de ejercer una parentalidad, no tiene que ver con cuestiones masculinas o femeninas. 
De acuerdo con Blas Radi (2018), cuando describe el mito n. ${ }^{\circ}$ 2, "los hombres trans no quieren tener hijxs":

El supuesto de la diferencia sexual (esto es, la creencia de que existen sólo dos tipos de cuerpos posibles, macho y hembra, y que a cada uno le corresponde uno y sólo un género, masculino y femenino, respectivamente) constituye todavía el currículum oculto de todas las disciplinas (incluida la educación sexual) y es la columna vertebral de nuestras instituciones. Así, es razonable pensar que el desinterés por la procreación pueda derivarse del funcionamiento normativo del género. En Argentina, los hombres trans no están obligados a renunciar a sus capacidades reproductivas para cambiar su documento. Así las cosas, el mito no pierde vigor. Su persistencia habilita la inexistencia de políticas de salud sexual y (no) reproductiva para esta población.

Adolescentes trans entre 14 y 18 años gestan en tasas semejantes a las de mujeres cis, según estudios de Light, Wang y Gomez-Lobo (2017). Por lo tanto, se torna necesario comprender mejor la fertilidad y posibilidades de cuidado de esas gestaciones, para que profesionales de la salud puedan atender las especificidades sexuales y (no) reproductivas de las personas trans. Es posible observar que diversas investigaciones consideran estas especificidades relacionadas, mínimamente, con cuatro áreas: biológica, social, psicológica y jurídica. Sin embargo, las investigaciones sobre gestaciones y abortos trans aún son escasas.

Mitu (2016) apunta que los procedimientos de transición física de género pueden causar esterilidad temporaria o permanente, pero que algunas tecnologías empleadas por la población trans posibilitan la reducción de esos impactos en la fertilidad y, de esa forma, eligen preservarla. Se encuentran investigaciones cualitativas con hombres y mujeres trans que utilizaron tecnologías reproductivas para preservar su fertilidad antes o durante su transición física. Por ejemplo, T’Sjoen, Van Caenegem y Wierckx (2013) hacen una contribución al discurso de las opciones reproductivas, basados en las experiencias de las personas trans y 
en una revisión bibliográfica sobre el desarrollo de nuevas técnicas de medicina reproductiva que preservan su fertilidad. En dicho estudio se discuten las posibilidades de preservar la fertilidad de los hombres trans y concluye que sí es posible mantenerla, aunque además de ser un tratamiento costoso y, por lo tanto, viable solo a una parte de esta población que tiene condiciones materiales de acceder, exige la interrupción del uso de hormonas.

La fertilidad está relacionada con la capacidad de gestar de los hombres trans y el deseo de conservar un cuerpo gestante. Según Wierckx et al. (2011), la terapia hormonal y quirúrgica de transgenitalización producen una pérdida irreversible del potencial reproductivo. Al respecto, el estudio investigó sobre el deseo reproductivo en hombres trans después de la intervención quirúrgica. Participaron 50 hombres trans, de los cuales 11 relataron tener hijes, ${ }^{2}$ esto es, el $22 \%$ de los participantes; 8 refirieron que no fue el cuerpo de ellos el que gestó sino el de la persona con la cual se relacionaban, en este caso, mujeres cis inseminadas por un banco de esperma; mientras que 3 de ellos atravesaron un proceso de embarazo y tuvieron un parto antes de la terapia hormonal y/o quirúrgica. Dicho estudio arrojó resultados interesantes al observar que, en el momento de la entrevista, más de la mitad de los participantes $(54 \%)$ deseaba una gestación para tener hijes. Concluye dicha investigación: los hombres trans que desean gestar, sugieren que es preciso más atención en la fase de diagnóstico de la transición y a las consecuencias sobre las posibilidades de gestación después de iniciada la terapia de transgenitalización.

Conforme lo observado, la posibilidad de gestar, atravesar un proceso de embarazo y someterse a una práctica de parto no es solo una realidad

2 Término que afirma un punto de vista plural, como modo alternativo al lenguaje masculino androcéntrico y hetero(cis)sexista. Con el uso de la "e", se coloca la indecibilidad del género en dos categorías reduccionistas y excluyentes, reivindicando la multiplicidad de experiencias sexo-genéricas que escapan a esa bicategorización de masculino y femenino (Theumer, 2018). 
presente en la vida de los hombres trans, sino que existen posibilidades de tornar cada vez más viable la fertilidad, aún después de la transición.

\section{Sobre la atención y los cuidados de la salud para los hombres trans}

Los estudios sobre la atención a la salud del hombre trans, especialmente en cuestiones relacionadas con la gestación y prácticas abortivas, son escasos. Las investigaciones encontradas, generalmente son del área de la salud, particularmente de medicina y especialmente de ginecología. En algunos de estos trabajos hay un reconocimiento de la precariedad en el conocimiento de esta población desde el punto de vista de las ciencias de la salud.

Wanta y Unger (2017), por ejemplo, partiendo de una revisión de la literatura sobre la salud de personas trans en la base Medline, buscaron identificar la situación de la práctica médica en relación con esa población y las necesidades de orientación en el contexto de los Estados Unidos. Según su estudio, la categoría médica ha estado en falta en cuestiones relacionadas con la investigación académica respecto a la población trans, resultando en lagunas de conocimiento para lidiar con los respectivos cuidados. En la búsqueda científica incluyeron artículos que atendieron a los criterios de inclusión, revisión, categorización y análisis en cuanto al contenido y delineamiento de la investigación. Identificaron 2405 artículos publicados desde el mes de enero de 1950 hasta junio de 2016, con foco en la salud de personas trans, principalmente en cirugía, salud mental y endocrinología. En los resultados señalan que aún hay lagunas significativas de conocimiento en subespecialidades, y apuntan limitaciones o dificultades que no se restringen al campo de la investigación o del conocimiento disponible, sino que abarcan la necesidad de ampliar el conocimiento para mejorar la atención a la salud. Entre las áreas no destacadas se pueden incluir los estudios en torno de cuestiones como el embarazo, ya que la práctica de la atención médica pone en evidencia la necesidad de una formación, 
especialmente en cuestiones de género y sexualidad, que se dedique a revisar la producción de normalidades y anormalidades. Es decir, y como señala Michel Foucault (2007), históricamente fue creándose un estatus de normalidad y de anormalidad que produjo condiciones para la marginalización, patología y persecución de toda existencia que no se adecuara a las normas sexo-genéricas. Esto, debido a una serie de mecanismos y prácticas sociales, médicas y jurídicas que crearon lo que es "natural” y la adecuación de los llamados "desvíos” como necesarios para ser considerado "saludable”. El concepto de anormal surge a finales del siglo XIX en el ámbito jurídico, y se traslada a la psiquiatrización del deseo y la sexualidad (Foucault, 2007). Entonces, es a partir de determinadas prácticas sociales estigmatizantes que va definiéndose a la transexualidad como una anormalidad.

Mientras siga vigente la patologización de las vidas que escapan a las normas que establecen un único modo de vivir las sexualidades, la formación de profesionales de la salud seguirá siendo deficiente y productora de sufrimientos para las personas trans. Por ejemplo, se observan dificultades en prácticas médicas en relación, especialmente, con la gestación de los hombres trans. Berger, Potter, Shutters y Imborek (2015), por ejemplo, señalan una dificultad inicial en el registro de una atención para hombres trans que se presentan para un diagnóstico prenatal, porque este sujeto y los proveedores del servicio estarán delante de un prontuario o formulario electrónico que designa, de modo binario y preestablecido, una configuración en femenino para documentar la gestación. Es este solo un ejemplo de cómo opera, sistemáticamente, una lógica binaria y excluyente para los hombres trans que acuden en busca de cuidados de salud, en este caso, obstétrica. En el estudio mencionado se apunta un déficit de literatura en medicina y enfermería que describa las experiencias en relación con el acceso a la salud de hombres trans gestantes.

En otro artículo sobre cuidados ginecológicos al hombre trans que gesta, Dutton, Koenig y Fennie (2008) reiteran que la reducida investigación 
y la escasa formación del personal encargado de prestar los servicios producen una situación de vulnerabilidad y precariedad, lo que genera indeseables consecuencias de violencia sistemática. De otra parte, según el estudio, los hombres trans tendrían un aumento en el riesgo de ser violentados sistemáticamente por el sistema de salud y de cometer suicidio. Investigaron 6 hombres trans en varios grados de transición social y médica e identificaron cuatro barreras principales en cuanto a la atención: 1) la importancia de la atención ginecológica, 2) los senos causaron el mayor conflicto de identidad de género; 3) el esfuerzo para revelar su identidad de género a los prestadores de cuidados de salud; y 4) el uso de pronombre por parte del equipo médico al momento de llenar los formularios de admisión en el servicio de salud.

Estas dificultades también son destacadas por Nixon (2013), quien señala cuestiones como fertilidad y barreras al acceso a la salud reproductiva. Subraya que se observa una reluctancia a ir a una clínica en busca de cuidados a la salud, e informa que más del 90\% de los participantes relató falta de información sobre su salud sexual, salud reproductiva, cuidados de aborto y atención prenatal.

Obedin-Maliver y Makadon (2016) también destacan la dificultad en la atención en salud en torno a las gestaciones trans, en los Estados Unidos. Según dicho estudio, muchos hombres trans mantienen sus órganos reproductivos y la capacidad de gestar. Las autoras destacan la necesidad de cuidados en diferentes fases, con consejerías previas a la concepción, con discusiones en torno a la interrupción de la testosterona cuando se desea gestar y durante el proceso de gestación. También sugieren más investigaciones sobre cómo fueron las experiencias con el parto y posparto para anticipar opciones sobre cómo amamantar y cuándo reiniciar la testosterona.

Shires y Jaffee (2015) señalan factores asociados a las experiencias de discriminación en cuidados de salud. Con base en un estudio con 1711 personas trans, les fue posible acceder a relatos sobre experiencias de 
acoso, violencias y discriminaciones en varios contextos, inclusive en el de cuidados de salud. En general, 41,8\% de los participantes narraron acoso verbal, agresión física o negación de igualdad en el tratamiento que recibieron en consultorios de clínicas y hospitales.

Dargie, Blair, Pukall y Coyle (2014), al explorar la identidad sexual y experiencias de personas trans, encontraron diversas identidades de género y orientaciones sexuales entre ellas. Por ejemplo, personas lésbicas, gays y bisexuales trans enfrentan barreras diferenciadas al acceder a los servicios de salud debido a la (in)visibilidad de sus identidades. De esa forma, un hombre trans puede enfrentar barreras significativas en el acceso a servicios ginecológicos, como un examen citológico. Como relata el argentino Tomás Máscolo a Verónica Dema (2018, párr. 2), no ha dado con profesionales de ginecología competentes:

No tuve buenas experiencias. Las visito cada tres meses, pero voy cambiando según la obra social, porque hasta el día de hoy no di con alguien que me trate bien, con quien me sienta seguro y cómodo hasta el final. Siempre hay alguna pregunta o trato incómodo. Cuando era más chico directamente no decía que era trans porque tenía mucho miedo.

Las insatisfacciones en relación con su condición de usuarios de los servicios de salud evidencian sensaciones de incomodidad, miedo e inseguridades. Como comenta Gian Franco Rosales en la misma nota de Dema (2018), publicada en un reconocido medio de comunicación argentino:

Es muy incómodo estar allí rodeado de mujeres que te miran y no entienden cómo es posible que un hombre vaya a ginecología (...) toda la consulta se basa en una entrevista donde el profesional pone el foco no en entender a quién tiene al frente para mejorar la atención, sino que la mayoría de las veces es para terminar la consulta diciendo: 'No estoy capacitado para atenderte', 'mi religión no me permite atender a personas como vos', 'primero tengo que derivarte a salud mental (Dema, 2018, párrs. 9 y 10). 
Barreras como estas, que tienen que ver con estigmas que derivan en situaciones discriminatorias, forman parte de un cotidiano de los hombres trans que buscan atender sus necesidades de salud y se encuentran con profesionales que aún se rigen por la lógica patologizante.

\section{Hombres trans gestantes: aspectos jurídicos, sociales y mediáticos}

Para entender cómo operan diferentes dispositivos a la hora de abordar el tema bajo estudio, es importante consultar un grupo de publicaciones relacionado con la gestación en hombres trans desde aspectos jurídicos, sociales y mediáticos. Karaian (2013) destaca la necesidad de una concepción de embarazo que no solo se dirija a mujeres cis, sino que abarque a todos los cuerpos con capacidad de gestar para que no les sean negados sus derechos reproductivos. Es decir, que no se puede reducir la experiencia de gestación solo a algunos cuerpos leídos como mujeres.

Riggs (2018) aborda la gestación trans en el contexto australiano y señala que existe una dinámica de poca atención debido a las políticas institucionales, a las especificidades de la población trans y, particularmente, a cuestiones relacionadas con la capacidad de gestar, parir y/o abortar. La investigación de Riggs se centra en cómo las personas gestantes son referidas en las leyes y las barreras de acceso a los servicios, situación que influye también en las limitaciones para mantener sus capacidades gestacionales.

Mientras que los movimientos sociales plantean diversos desafíos en cuanto a la urgencia de una escucha atenta a las demandas específicas de la población trans, se avanza en el sentido de comprender algunos aspectos sociales que afectan a los hombres trans que atraviesan por procesos de gestación. Motmans (2011), por ejemplo, se refiere al impacto del movimiento trans belga en favor del bienestar de dicha población. En su estudio destaca el esfuerzo exitoso de la comunidad trans por construir una plataforma que junte las voces que dialogan 
para posicionar y llamar la atención respecto a estos asuntos. En la mencionada plataforma aparecen temas como la irreversibilidad de la pérdida de fertilidad en hombres trans, por ejemplo.

En España también ha sido destacada la importancia de los movimientos sociales en torno a los derechos de la comunidad trans (Platero, 2011), incluyendo nuevos desarrollos en la agenda cultural, social y política de ese contexto. En ese sentido, los principales problemas que envuelven cuestiones de ciudadanía, también se traducen en derechos sexuales, como políticas de orientación sexual y de identidad de género, acceso a información y autoconocimiento del propio cuerpo; derechos reproductivos, como reproducción asistida, métodos contraceptivos, acceso al aborto seguro; y derecho a la asociación, como matrimonio igualitario, derecho al divorcio, entre otros.

Otra dimensión que viene siendo analizada se refiere a la exposición de los hombres trans gestantes en los medios de comunicación. Verlinden (2012), al tratar el tema de cuerpos trans gestantes, hace referencia a diversos eventos mediáticos que llamaron la atención respecto al tema desde el año 1990, abriendo debates que colaboraron para establecer condiciones de posibilidad de visibilización de las experiencias para la población trans. Currah (2008) también se refiere al impacto en los medios del "hombre embarazado", en especial el caso de Thomas Beatie, un hombre trans que se sometió a una cirugía de transgenitalización y se encontraba en proceso de tratamiento con terapia hormonal, pero había parado de tomar testosterona antes de gestar. El autor narra cómo el hecho fue recibido con una mezcla de curiosidad y repulsión, aborrecimiento, indiferencia y conmemoración. Algunos blogs expresaban que, por el hecho de Thomas estar embarazado, sentían que "ella era aún una mujer”; otros creían que la transición debería significar que él había "perdido el derecho a gestar"; algunos blogs de mujeres expresaron aborrecimiento por toda la atención que un "hombre embarazado" estaba recibiendo; y una pequeña proporción parecía no tener problemas para entender y aceptar comúnmente esas experiencias. 
Pérez Navarro (2017) se refiere a la presencia en los medios de historias de algunos hombres trans que tomaron la decisión de interrumpir temporalmente sus tratamientos hormonales para poder gestar. Cuenta que ellos optaron por una gestación de forma pública, concediendo entrevistas y permitiendo la divulgación de imágenes en los medios de comunicación sobre sus propios cuerpos gestantes y gestando, generando con ello innumerables críticas tanto desde la comunidad trans como desde perspectivas del transodio. ${ }^{3}$ Las críticas basadas en estructuras cisheteronormativas recibieron amplia cobertura en la prensa, en programas de TV, fórums en Internet y otros medios.

Referencias más recientes retoman la cuestión de la divulgación mediática de la gestación trans. Andal (2019) examinó las selfies (autofoto) de hombres trans embarazados, así como su creciente visibilidad pública y cómo esas gestaciones se enfrentan al discurso moral y de patologización de sus cuerpos. El autor argumenta que la exhibición visual de estos cuerpos no es una forma de alteridad o reconocimiento, sino una intencionalidad de suspensión de la moralización y patologización de las identidades trans. La gestación transexual tiene la característica de interrumpir el concepto de embarazo comúnmente pensado o imaginado y, al mismo tiempo, evoca una experiencia muy familiar de reproducción.

Otro elemento que viene llamando la atención sobre las gestaciones de hombres trans en los medios de comunicación es la producción de documentales. Pearce y White (2019) hacen un breve análisis de uno de esos documentales. A Deal With The Universe (Un acuerdo con el universo) cuenta la historia de cómo Jason Barker enfrenta el parto de su hijo. Una imagen promocional del documental, utilizada especialmente para su lanzamiento más amplio en el cine del Reino Unido, en 2019, muestra de forma destacada a Barker embarazado en una piscina.

3 Se utiliza aquí el término "transodio" como una forma de destacar la dimensión estructural y estructurante del sistema cisheteronormativo que dirige el odio hacia lo que transborda de este. 
Como gran parte del lenguaje escrito y visual utilizado para dignificar las gestaciones trans en los medios, esas imágenes son producidas y se muestran en una yuxtaposición de características corporales normativamente asociadas a la producción de femineidad y masculinidad.

\section{Aborto para los hombres trans: algunas consideraciones}

Hablar sobre cuerpos gestantes implica, también, conocer sobre las características (no) reproductivas de los hombres trans. Es decir, sobre la posibilidad de gestar y, por lo tanto, de acceder a una interrupción de esa gestación. Como ha señalado Judith Butler (2016), en su paso por la Argentina, no todos los sujetos sexuados son reproductivos; hay personas que no tienen edad reproductiva, algunas personas jamás serán capaces de reproducirse, otras jamás querrán reproducirse, y muchas viven su vida sin reproducirse; entonces, dada la gran multiplicidad de posiciones corpóreas en relación con este mandato cultural de reproducirse, ise puede decir que es necesario u obligatorio éticamente concebir al cuerpo sexuado fuera de los términos de la reproducción? Después de todo, la reproducción sexual es solo una manera de organizar y entender la sexualidad del cuerpo. Es por ello que se considera la práctica abortiva como posibilidad en cuerpos trans, y, de acuerdo con Blas Radi (2018, Mito n. ${ }^{\circ}$ 6), "los hombres trans sí pueden embarazarse! Muchos lo hacen y algunos de ellos abortan”, como puede percibirse también en la narrativa de Tomás Máscolo:

En mi vida aborté dos veces. La primera lo hice solo, en una "clínica” que era un garaje en la zona sur de Rosario. Junté la plata como pude. Aún recuerdo la madera que atravesaba la camilla y los pañales con los que fui despedido 30 minutos después de la intervención. La segunda, fue con Misoprostol. Todavía recuerdo los dolores de parto en mi casa.

Lejos de intentar reproducir una mirada victimizante, hay que dejar en claro que el aborto es la última consecuencia de una cadena de violencia. La Ley de Educación Sexual Integral no contempla que los cuerpos de los 
hombres trans muchas veces son fértiles. A eso se le suma el desdén de médicos que escogen la violencia y discriminación a un cuerpo que "no es normal”. (2017, párr. 3).

Es altamente preocupante el déficit de investigaciones relacionadas con el aborto de los hombres trans. Esto, tiene que ver, también, con motivos ligados a la producción de criminalidad en torno a la práctica, presente aún en muchos países del mundo. Según un estudio cualitativo realizado en Argentina sobre esta cuestión, en el que se entrevistaron a hombres trans activistas, es alarmante la necesidad de inclusión de los hombres trans en los debates sobre el acceso a la interrupción de la gestación (Nichols, 2018). Según el estudio, en el ámbito legal en el marco de la Ley de Identidad de Género, se provee el lenguaje para una noción más amplia de género, lo cual ofrece una base jurídica que funciona en contra de la reducción del sujeto de los derechos sexuales y no reproductivos solo a las mujeres cis. No obstante, y siguiendo con los resultados de la mencionada investigación, ese cambio no es suficiente para significar la mejor inclusión de hombres trans en los debates sobre acceso a prácticas seguras de aborto. Se necesita una participación más efectiva de ellos, afirma la autora.

"El aborto en hombres trans es una problemática que todavía está abordada de forma muy incipiente en la salud pública. Las estadísticas son cuasi nulas con respecto a esta población (...) de nosotros... no se habla” (Máscolo, 2017, La igualdad). Olivia Nichols (2018) destaca la importancia de los movimientos sociales como agentes necesarios para cuestionar a las instituciones que violentan y excluyen a los hombres trans, incluyéndose la pauta del aborto como central para garantizar sus derechos sexuales y (no) reproductivos. Como nos recuerda Blas Radi (2018, Mito n. ${ }^{\circ}$ 6):

Hay personas que no son mujeres que de hecho se embarazan y abortan -los hombres trans, por ejemplo-. Sin embargo, el mito recurre a una idea de humanidad que supone que todas las personas (dignas de atención, al menos) son cis: es decir, una concepción cisexista de la humanidad. 
Radi aborda también la necesidad de incluir en la agenda de pesquisa y de los movimientos sociales la temática de salud sexual y (no) reproductiva de los hombres trans. Así mismo, llama la atención sobre la producción de estadísticas en relación con investigaciones que evidencian grandes limitaciones. Como se mencionó al comienzo de este artículo, las investigaciones sobre los hombres trans y sus derechos (no) reproductivos no arrojan números significativos, si se comparan con la cantidad de estudios respecto a mujeres cis. Según el análisis de Radi (2018), cuando se interroga sobre los procedimientos para determinar estas cifras, los cálculos realizados y los datos brutos, se observa que se trata de investigaciones que son solamente aplicadas a mujeres cis, subentendiendo que son solo esos los cuerpos con capacidad de gestar, parir y/o abortar, por ejemplo. El autor afirma que la inexistencia de hombres trans en estos asuntos no es un resultado de la investigación, sino consecuencias de una premisa. En esas circunstancias, se hace preciso considerar que probablemente esa mayoría absoluta se deba a una serie de limitaciones que pertenecen a los instrumentos de análisis que son implementados para abordar estas temáticas. El investigador señala, también, que aún si existiesen herramientas analíticas más elaboradas, capaces de abordar y registrar a los hombres trans, sería necesario considerar un conjunto de desafíos antes de pensar en los números. Por ejemplo, considerar las violencias sistemáticas a las que los hombres trans se enfrentan, y por las cuales ellos no estén dispuestos a identificarse públicamente en un centro de salud. Y cuando de abortos se trata, entonces los desafíos se multiplican, advierte.

La Investigación sobre aborto en América Latina y el Caribe. Una agenda renovada para informar políticas públicas e incidencia, cuyo resumen ejecutivo fue publicado por Silvina Ramos (2016), nos ofrece un conocimiento riguroso y sólido de la evidencia científica de que se dispone en la región sobre este tema en el periodo de 2009 al 2014. Dicha investigación realiza algunos señalamientos para una agenda renovada en cuanto a informar políticas públicas e incidencia; sugiere que es necesario y urgente disminuir las brechas de investigación sobre los 
hombres trans y el aborto; plantea como "estudios a futuro", la necesidad de reforzar las perspectivas de género en términos "relacionales"; e indica que precisamos propuestas para investigar más sobre las experiencias del aborto de los hombres trans cuya capacidad gestante está intacta y, por ende, pueden necesitar interrumpir una gestación. Mario Pecheny, autor del capítulo de esta misma investigación titulado "Varones y aborto. Acompañarlas en lo que ellas decidan: aborto y participación de los hombres”, propone investigar la participación o la alianza de varones gays, cis, trans y heterosexuales en los movimientos sociales que pugnan por el derecho al acceso al aborto seguro y la participación de los hombres trans en dichas reivindicaciones.

Peralta (2015) aborda, desde una perspectiva jurídica, situaciones que afrontan gays, lesbianas, transexuales y bisexuales respecto a su reconocimiento como familia y sus posibilidades de tener hijos e hijas, ya sea utilizando las nuevas tecnologías de reproducción o no. La autora señala que aportar los espermatozoides para una fecundación, no convierte a una persona en padre, o madre, si esa persona es una travesti o una mujer trans. De la misma manera, si un hombre trans lleva adelante su gestación, con óvulos propios o no, eso no lo convierte en padre. La investigadora afirma que las relaciones sexuales que pueden resultar en una gestación, independientemente de la orientación sexual y la identidad de género, también pueden requerir un aborto. Esto nos coloca, nuevamente, ante un escenario de déficit de investigaciones detalladas sobre el tema del aborto trans.

Bergallo, Jaramillo y Vaggione (2018) señalan que es urgente la necesidad de un tratamiento exhaustivo en el debate sobre el aborto y las identidades de género. Su obra aborda y problematiza, desde una perspectiva regional, distintas dimensiones relevantes en el debate por la legalización del aborto. Destacan la necesidad de abordaje del aborto de personas trans en un contexto de creciente reconocimiento a la identidad de género en varios países de la región, para comprender el pluralismo sociológico y legal del debate. Se afirma que ese reconocimiento 
ha implicado un proceso de visibilización sobre el hecho de que la capacidad de gestar y, por ende, de abortar, no solo atañe a las mujeres cis. Y, en ese sentido, según Olivia Nichols (2018, p. 14), “desafortunadamente este binarismo biologicista todavía persiste en varios espacios del movimiento por el aborto legal en Argentina”. Afirmación sustentada en la entrevista realizada a Tomás Máscolo (2018), quien manifestó:

Participar en los talleres por el aborto legal en estos encuentros como activista trans siempre fue una experiencia negativa porque, por un lado, un sector del feminismo le decía que él había traicionado a la lucha por elegir "la identidad del patriarcado" y por el otro lado, otro sector le decía que participe sino como mujer "porque tengo vagina (Nichols, 2018, p. 14).

Narrativas como la de Tomás se hacen presentes mostrándonos cómo opera el binarismo sexo-genérico a la hora de marcar los cuerpos que gestan, paren y abortan. Los hombres trans que abortan, además del contexto restrictivo de normativas que criminalizan la práctica del aborto, se enfrentan con dificultades que atañen a la propia identidad de género, sea en el ámbito de la salud y los obstáculos que mencionan varios de los estudios citados, sea en el activismo en los movimientos sociales, cuando aún operan los binarismos biologicistas al reivindicar un sujeto político del aborto, en estos casos, a la mujer cis, por ejemplo. Los hombres trans crean sus narrativas:

Después de terminar una relación de dos años con una travesti, descubrió que estaba embarazado. Solo, y sujeto a la vulnerabilidad, sin siquiera pasar por todas las transformaciones en el cuerpo, él decidió abortar. Lo hizo por medio de métodos que colocaron en riesgo su salud. (NLucon, 2010, párr. 2, traducción libre).

En este caso, una relación heterosexual entre un hombre trans y una travesti nos muestra qué tan frágiles son algunas formas de entender el género y la sexualidad. Experiencias que escapan y dicen de otras experiencias; y son esas experiencias sobre la gestación y al aborto 
transgénero las que nos colocan frente a irrupciones respecto a las normas sexo-genéricas, y nos exigen urgentemente avanzar en materia de investigaciones relacionadas con el aborto de los hombres trans.

\section{Consideraciones finales}

Para efectuar una aproximación al conocimiento producido en relación con las experiencias de procesos de gestación y aborto de los hombres trans, se realizó una revisión de la literatura como herramienta de información para invitar a la discusión sobre este tema en el ámbito académico. Considerada en sí misma una de las primeras etapas del proceso de investigación (Guirao Goris, 2015), esta revisión de literatura auxiliaría para identificar cuánto se conoce y se desconoce sobre el tema que aquí ocupa, y de esa forma obtener una idea sobre el estado actual de la producción de conocimiento académico en relación con dicha cuestión.

Esta revisión implicó una valoración crítica de las investigaciones en este contexto, para ofrecer un panorama objetivo del conocimiento sobre este tema particular, a saber: el proceso de gestación y aborto de hombres trans.

Se observa, como fue resaltado anteriormente, un déficit de investigaciones que consideren y destaquen el valor narrativo de las experiencias en primera persona, sobre procesos de gestación, parto y, particularmente, aborto de las personas trans. Diversas controversias atraviesan esta práctica, y esa preocupación guía, de alguna forma, hacia un ejercicio ético para pensar sobre qué abortos son esos que quedan al margen de la discusión, cuando pautamos el tema. Importante destacar que "hombres trans y personas transmasculinas, como aquellas que no responden a ninguna categoría, sangran, gestan y se practican abortos" (Barone, 2018, p. 103, traducción libre). Las existencias que sangran, menstrúan, gestan y abortan, son las que nos invitan a cuestionar nuestras propias caídas en actualizadas trampas que producen discursos que excluyen, inclusive, en la agenda de los movimientos sociales. 
En esta revisión de literatura se hace evidente que, particularmente, la práctica abortiva de los hombres trans es una problemática que aún es abordada de forma muy incipiente. Por ello, investigar sobre el aborto es entenderlo como una práctica, y, por lo tanto, como alteridad. De esa forma, se abre la posibilidad de pensar la práctica abortiva fugándose de las lógicas de la reproducción heterocisnormativa. Si nos proponemos, en cada espacio de actuación, habilitar una escucha activa que recupere un régimen de sensibilidad múltiple y polifónica, otras realidades sobre aborto se harán presentes, sea en la ruta de atención a la salud sexual de los hombres trans, en el movimiento social, en la academia o en la cotidianeidad de las relaciones interpersonales.

Se considera, como un tema relevante para la coyuntura latinoamericana y del Caribe con respecto a las políticas sobre el acceso al aborto, que es de extrema importancia disminuir las brechas de investigación sobre los hombres trans y esta práctica, para que esas vidas no se vean restringidas sexual y (no) reproductivamente. Es necesario y urgente colocarnos en la investigación y en la comprensión sobre las experiencias de las personas trans en cuanto a su carácter de personas gestantes.

\section{Referencias}

Almeida, Guilherme. (2012). 'Homens trans': novos matizes na aquarela das masculinidades? Revista. Estudos Feministas, 20(2), 513-523.

Andal, Aireen Grace. (2019). Self-Shooting Uterus-Owners: Examining the Selfies of Pregnant Transmen within the Politics of Human Reproduction. Changing Societies \& Personalities, 3(1), 36-51.

Barone, María Antonella. (2018). Senhoras De Si: Problematizando as incidências das biopolíticas nos corpos que sangram e co-produzindo narrativas que (re)inventam a vida a partir da prática do aborto (Dissertação de mestrado). Universidade Federal do Espirito Santo (UFES), Vitória, Espirito Santo, Brasil.

Bento, Berenice. (2008). O que é transexualidade. São Paulo: Editora Brasiliense. 
Bento, Berenice. (2014). A reinvenção do corpo: sexualidade e gênero na experiência transexual (Segunda ed.). Natal, RN, Brasil: Editora da Universidade Federal do Rio Grande do Norte.

Bento, Berenice. (2017). Transviad@s: gênero, sexualidade e direitos humanos. Salvador: Editora da Universidade Federal da Bahia.

Bergallo, Paola; Jaramillo, Isabel y Vaggione, Juan (Comp.). (2018). El aborto en América Latina. Estrategias jurídicas para luchar por su legalización y enfrentar las resistencias conservadoras. Buenos Aires: Siglo XXI Editores. https://www.cmi.no/publications/file/6584-movimiento-transnacionalcontra-el-derecho-al.pdf

Berger, Anthony; Potter, Elizabeth; Shutters, Christina y Imborek, Katherine. (2015). Pregnant transmen and barriers to high quality healthcare. Proceedings in Obstetrics and Gynecology, 5(2), 1-12.

Butler, Judith. (2015). Cuerpos que aún importan. (Conferencia dictada en la Universidad Nacional de Tres de Febrero, Argentina). Recuperado de https://www.youtube.com/watch?v = -UP5xHhz17s

Castro-Peraza, María-Elisa de; García-Acosta, Jesús; Delgado-Rodriguez, Naira; Sosa-Alvarez, María; Llabrés-Solé, Rosa; Cardona-Llabrés, Carla y LorenzoRocha, Nieves. (2019). Biological, Psychological, Social, and Legal Aspects of Trans Parenthood Based on a Real Case-A Literature Review. International Journal of Environmental Research and Public Health, 16(6), 925.

Currah, Paisley. (2008). Expecting bodies: The pregnant man and transgender exclusion from the employment non-discrimination act. Women's Studies Quarterly, 36(3/4), 330-336.

Charter, Rosie; Ussher, Jane; Perz, Janette \& Robinson, Kerry. (2018). The transgender parent: Experiences and constructions of pregnancy and parenthood for transgender men in Australia. International Journal of Transgenderism, 19(1), 64-77.

Dargie, Emma; Blair, Karen; Pukall, Caroline \& Coyle, Shannon. (2014). Somewhere under the rainbow: Exploring the identities and experiences of trans persons. The Canadian Journal of Human Sexuality, 23(2), 60-74.

Dema, Verónica. (4 de septiembre de 2018). Varones trans y la incomodidad en los consultorios ginecológicos. Recuperado en julio de 2020, de: https:// www.lanacion.com.ar/sociedad/varones-trans-incomodidad-consultoriosginecologicos-nid2166704 
Dutton, Lauren; Koenig, Karel \& Fennie, Kristopher. (2008). Gynecologic care of the female-to-male transgender man. Journal of Midwifery \& Women's Health, 53(4), 331-337.

Ellis, Simon; Wojnar, Danuta \& Pettinato, Maria. (2015). Conception, pregnancy, and birth experiences of male and gender variant gestational parents: it's how we could have a family. Journal of Midwifery \& Women's Health, 60(1), 62-69.

Foucault, Michel. (2007). Los anormales (Primera ed., cuarta reimpresión). Buenos Aires: Fondo de Cultura Económica.

García Becerra, Andrés. (2009). Tacones, siliconas, hormonas y otras críticas al sistema sexo-género. Feminismos y experiencias de transexuales y travestis. Revista Colombiana de Antropología, 45(1), 119-146.

Guirao Goris, Silamani J. Adolf. (2015). Utilidad y tipos de revisión de literatura. Ene, 9(2). Recuperado de: https://dx.doi.org/10.4321/S1988$348 \times 2015000200002$

Hoffkling, Alexis; Obedin-Maliver, Juno y Sevelius, Jae. (2017). From erasure to opportunity: a qualitative study of the experiences of transgender men around pregnancy and recommendations for providers. BMC Pregnancy and Childbirth, 17(2), 332.

Johnson, Emilie \& Finlayson, Courtney. (2016). Preservation of fertility potential for gender and sex diverse individuals. Transgender Health, 1(1), 41-44.

Karaian, Lara. (2013). Pregnant Men: Repronormativity, Critical Trans Theory and the Re(conceive)ing of Sex and Pregnancy in Law. Social \& Legal Studies, 22(2), 211-230.

Light, Alexis; Obedin-Maliver, Juno; Sevelius, Jae \& Kerns, Jennifer. (2014). Transgender men who experienced pregnancy after female-to-male gender transitioning. Obstetrics \& Gynecology, 124(6), 1120-1127.

Light, Alexis; Wang, Lin-Fan \& Gomez-Lobo, Veronica. (2017). The Family Planing Needs of Young Transgender Men. Journal of Pediatric \& Adolescent Gynecology, 30(2), 274.

Máscolo, Tom. (28 de septiembre de 2017). Soy hombre trans y aborté: nadie sabe qué hacer con nuestros cuerpos. Recuperado de: http://agenciapresentes.org/2017/09/28/hombre-trans-aborte-nadie-sabe-cuerpos/ 
Mitu, Khadija. (2016). Transgender Reproductive Choice and Fertility Preservation. AMA Journal of Ethics, 18(11), 1119-1125.

Motmans, Joz. (2011). 'We Can’t Have Men Giving Birth!' (But We Do): The Impact of the Belgian Transgender Movement on the Well-Being. In Alison Woodward, Jean-Michel Bonvin \& Mercè Renom (Eds.), Transforming Gendered Well-Being in Europe: The Impact of Social Movements (pp. 169-186). Burlington, VT: Ashgate.

Nichols, Olivia. (2018). Arguing from within: The Marginalization of Trans Men within the Movement for Legal Abortion in Argentina. Recuperado de: https://digitalcollections.sit.edu/isp_collection/2891

Nixon, Laura. (2013-2014). The Right to (Trans) Parent: A Reproductive Justice Approach to Reproductive Rights, Fertility, and Family-Building Issues Facing Transgender People. William \& Mary Journal of Race, Gender, and Social Justice, 20(1).

Lucon, NLucon. (8 de dezembro de 2016). Homens trans debatem a descriminalização do aborto no Brasil. Recuperado en mayo de 2019, de: http:// paradiversidade.com.br/2010/2016/12/10/15108/

Obedin-Maliver, Juno \& Makadon, Harvey. (2016). Transgender men and pregnancy. Obstetric medicine, 9(1), 4-8.

Pearce, Ruth \& White, Francis. (2019). Beyond the pregnant man: representing trans pregnancy in A Deal With The Universe. Feminist Media Studies, 19(5), 1-3.

Peralta, María Luisa. (2015). Lxs niñxs en las familias GLTB: un panorama de la situación actual. Revista Niños, Menores e Infancias, (10). ISSN 1668-8570.

Pérez Navarro, Pablo. (2017). On ne naît pas queer: From The Second Sex to Male Pregnancy. In Andrea Duranti \& Matteo Tuveri (Eds.), Proceedings of the 18th Conference of the Simone de Beauvoir Society: Yesterday, Today and Tomorrow (pp. 327-337). Newcastle upon Tyne, UK: Cambridge Scholars Publishing.

Platero, Raquel. (2011). The narratives of transgender rights mobilization in Spain. Sexualities, 14(5), 597-614.

Radi, Blas. (29 de mayo de 2018). Mitología política sobre aborto y hombres trans. Recuperado en julio de 2020, de: https://sxpolitics.org/es/3945$2 / 3945$ 
Ramos, Silvina. (2016). Investigación sobre aborto en América Latina y el Caribe. Una agenda renovada para informar políticas públicas e incidencia (resumen ejecutivo). Estudios demográficos y urbanos, 31(3), 833-860. Recuperado el 24 de septiembre de 2019, de: http://www.scielo.org.mx/ scielo.php?script $=$ sci_arttext\&pid $=$ S0186-72102016000300833\&lng $=$ es\&tl $\mathrm{ng}=\mathrm{es}$

Riggs, Damien. (2018). Trans Pregnancy. An International Exploration of Transmasculine Practices of Reproduction. Law and Policy Review Australia. Flinders University.

Shires, Deirdre \& Jaffee, Kim. (2015). Factors associated with health care discrimination experiences among a national sample of female-to-male transgender individuals. Health \& Social Work, 40(2), 134-141.

Theumer, Emanuel. (2018). Lenguaje incisivo. Blog Parole de queer. Recuperado de http://paroledequeer.blogspot.com/2018/09/lenguaje-incisivoemmanuel-theumer.html

T'Sjoen, Guy; Van Caenegem, Eva \& Wierckx, Katrien. (2013). Transgenderism and reproduction. Current Opinion in Endocrinology, Diabetes and Obesity, 20(6), 575-579.

Veale, Jaimie; Watson, Ryan; Adjei, Jones \& Saewyc, Elizabeth. (2016). Prevalence of Pregnancy Involvement Among Canadian Transgender Youth and its Relation to Mental Health, Sexual Health, and Gender Identity. International Journal of Transgenderism, 17(3-4), 107-113.

Verlinden, J. (2012). Transgender Bodies and Male Pregnancy: The Ethics of Radical Self-Refashioning. In Michaela Hampf \& MaryAnn Snyder-Körber (Eds.), Machine: Bodies, Gender, Technologies (pp. 107-136). Heidelberg: Universitätsverlag Winter.

Wanta, Jonathon \& Unger, Cecile. (2017). Review of the transgender literature: where do we go from here? Transgender Health, 2(1), 119-128.

Wierckx, Katrien; Van Caenegem, Eva; Pennings, Guido; Elaut, Els; Dedecker, David; Van de Peer, Fleur ... \& T’Sjoen, Guy (2011). Reproductive wish in transsexual men. Human Reproduction, 27(2), 483-487. 